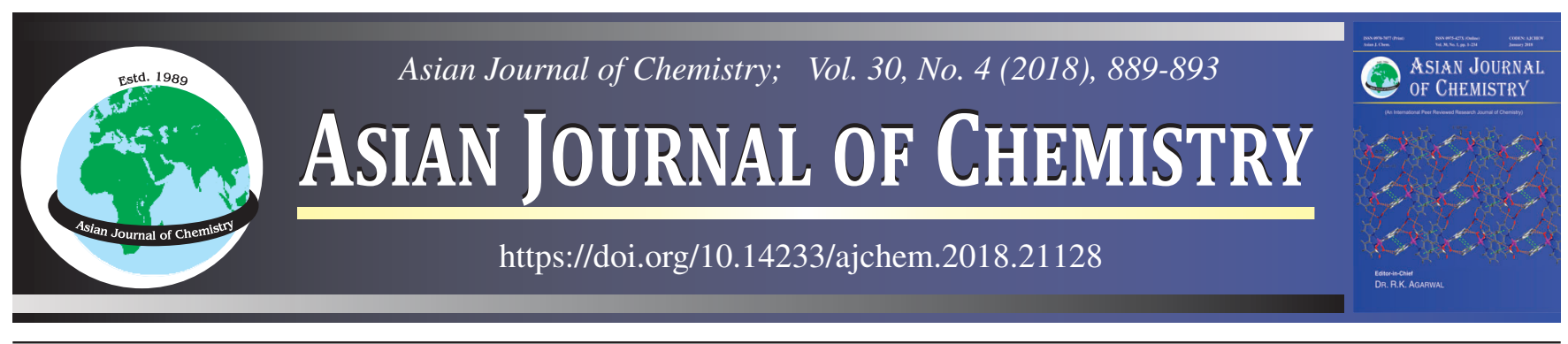

\title{
Optimization of RP-Gradient-HPLC Method for Evaluation of Impurities of Scopolamine from Transdermal Delivery Patch
}

\author{
Galla Vamsi Krishna ${ }^{1, *}$, T. Krishna Murthy ${ }^{1}$ and J. RaJeswari ${ }^{2, *}$
}

${ }^{1}$ Analytical Research and Development, Generics, IPDO, Dr. Reddy's Laboratories, Hyderabad-500 049, India

${ }^{2}$ Department of Biochemistry, Acharya Nagarjuna University, Guntur-522 510, India

*Corresponding authors: E-mail: vamsikg@drreddys.com; jinkarajeswari@gmail.com

A new reversed phase gradient-HPLC method is developed for evaluation of impurities of scopolamine from transdermal delivery patch formulation, prescribed to treat addiction-related disorders. Selection of stationary phase and chromatographic conditions were discussed regarding separation efficiency and gradient consistency of the newly developed method. Challenges related to extraction of impurities along with active ingredient from inactive polymer matrix which contain multiple layers and coatings were fully addressed. The method is developed by using Inertsil ODS-3V column with phosphate buffer ( $\mathrm{pH} 5.5$ ) as aqueous phase and combination of acetonitrile and methanol as organic modifier. At flow rate of $0.7 \mathrm{~mL} / \mathrm{min}$ with linear gradient, scopolamine and its related impurities namely Imp-A, B, C and $\mathrm{D}$ were monitored at the wavelength of $210 \mathrm{~nm}$. The finalized chromatographic conditions and test methodology is verified as per ICH Q2 (R1) guideline. Linearity and accuracy of the method was found to be within the range of LOQ to $150 \%$ with correlation coefficient (r) of $0.999 \pm 5 \%$.

Keywords: Scopolamine, Impurities, Gradient-HPLC, Transdermal delivery system, Method validation.

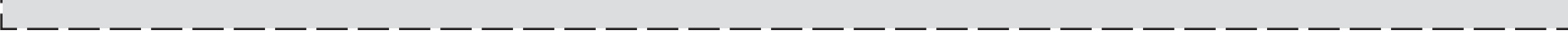

\section{INTRODUCTION}

Beside oral, intravenous, intramuscular, topical and other route of administrations, transdermal delivery system (TDS) provides a controlled release of the medication into the patient, usually through either a porous membrane covering a reservoir of medication or through body heat melting thin layers of medication embedded in the adhesive. Transdermal delivery system can surpass first-pass metabolism of drugs by liver, enhanced bioavailability and efficacy. Furthermore, transdermal delivery system improves patient's compliance by providing painless, non-invasive and self-administrable dosage forms. Recent advancements in pharmaceutical sectors achieved successful delivery of antihypertensive, antidepressant, vitamin, hormone therapy and pain management drugs in form of transdermal patches.

One of such transdermal delivery system, the first commercially available prescription patch approved by the U.S. FDA is scopolamine, an anticholinergic alkaloid with medical applications in very minute doses, used to treat addiction-related disorders. Scopolamine is used to relieve nausea, vomiting and dizziness associated with motion sickness and recovery from anaesthesia and surgery. Scopolamine may also be used in the treatment of Parkinson's disease, spastic muscle states, irritable bowel syndrome, diverticulitis and other conditions. Due to its multiple pharmacological advantages, scopolamine is one among World Health Organization's list of essential medicines, the most effective and safe medicines needed in a health system. Scopolamine is a viscous liquid that has a molecular weight of $303.35 \mathrm{D}$ and its chemical fortmula is $\alpha$-(hydroxymethyl)benzeneacetic acid 9-methyl-3-oxa-9-azatricyclo [3.3.1.02,4]-non-7-yl ester. The empirical formula is $\mathrm{C}_{17} \mathrm{H}_{21} \mathrm{NO}_{4}$ and has pKa of 7.55-7.81. Each transdermal delivery patch contains $1.5 \mathrm{mg}$ of scopolamine as base, is a circular flat patch designed for continuous release following application to an area of intact skin on the head, behind the ear [1].

Few articles were published which describes about extraction and analysis of scopolamine along with its derivatives, tropines from natural sources and medicinal plants using liquid chromatography methods [2-9]. Few works described about analysis of scopolamine and its degradation products by GLC, GC and GC-MS [10-12]. The works also described about stress studies, pharmacokinetic applications and analysis of scopolamine from rat and human plasma by LC-MS and MS-MS [13-16]. Some more works details about the analysis of scopolamine using conventional ways like densitometic, potentiometric and radio-receptor based techniques [17-19]. As per literature survey, no work till date is available regarding impurities 
of scopolamine from drug products. Hence, our current study is focused on the evaluation of impurities of scopolamine especially from transdermal patch which is a novel drug delivery system using gradient HPLC, where till date no work is published and not official in any pharmacopia and is first of its kind. Method development is performed for separation of 4 known impurities and trials regarding selection and screening of chromatographic conditions, diluent and extraction procedures were well discussed. Feasibility, suitability of newly developed method is verified as per ICH.

\section{EXPERIMENTAL}

Standards (>98\%) and samples of scopolamine and its 4 impurities namely Impurity-A, B, C and D (>98 \%) (Fig. 1) were supplied by Dr. Reddy's laboratories limited, Hyderabad, India. ACS-Emsure grade (> $99 \%)$ potassium dihydrogen phosphate, potassium hydroxide, gradient grade (>99\%) methanol, acetonitrile and acetone were procured from Merck, Germany. High purity, deionized water filtered through $0.22 \mu$ membrane filter is obtained from Millipore Milli-Q Plus water purification system (Millipore, Milford, MA, USA).

Equipment: HPLC system (Model: Alliance 2695, Make: Waters, Milford, USA) is used, consists of a quaternary pump, auto sampler and a photo-diode array detector (PDA 2998). The output signal is monitored and processed using empower3 software. Sonicator, Rotary shaker (Labtech, Korea) were used during mobile phase and sample preparation. Photo stability studies were carried out in a photo stability chamber (Sanyo,

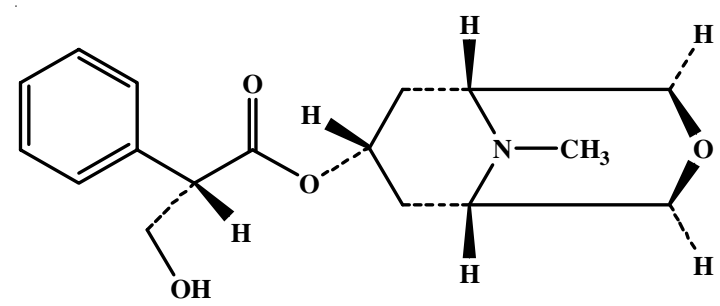

Scopalamine

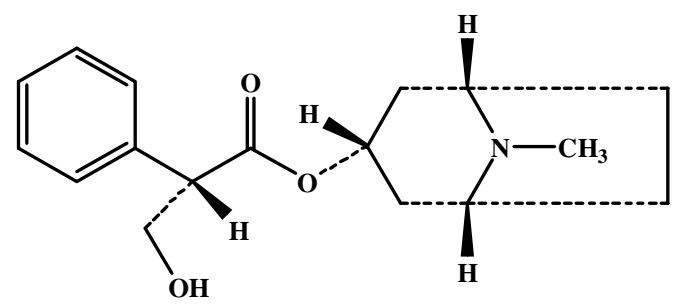

Imp-A (Hyoscyamino)

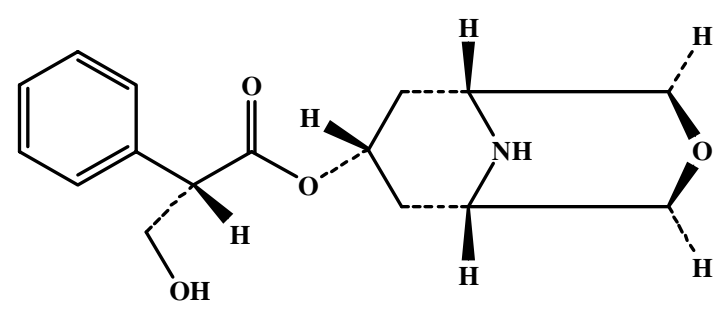

Imp-B (Norhysocine)
UK) and thermal stability studies were performed in a dry air oven (Thermolab, India).

Chromatographic conditions: The method is developed using Inertsil ODS-3 V, $250 \mathrm{~mm} \times 4.6 \mathrm{~mm}, 5 \mu \mathrm{m}$ column. Buffer is prepared with potassium dihydrogen phosphate $(25 \mathrm{mM})$, $\mathrm{pH}$ adjusted to 5.5 with potassium hydroxide and filtered through $0.45 \mu$ membrane filter. Mobile phase-A is prepared by mixing buffer-acetonitrile in ratio of $95: 5, \mathrm{v} / \mathrm{v}$ and mobile phase- $\mathrm{B}$ is prepared by mixing methanol-acetonitrile-water in ratio of 40:40:20, v/v/v. The gradient program (time in $\mathrm{min} /$ $\% \mathrm{~B}$ ) is set as $0.0 / 5,10.0 / 5,30.0 / 35,40.0 / 85,40.1 / 5,45.0 / 5$ with flow rate of $0.7 \mathrm{~mL} / \mathrm{min}$. The column oven temperature is maintained at $45^{\circ} \mathrm{C}$ and the injection volume is $50 \mu \mathrm{L}$. Scopolamine and its related impurities were monitored at UV wavelength of at $210 \mathrm{~nm}$.

Preparation of diluted standard solution: A mixture of acetone and Milli-Q water in ratio of 10:30, v/v is used as diluent. A primary stock solution of $1 \mathrm{mg} / \mathrm{mL}$ is prepared by dissolving appropriate amount of scopolamine standard in diluent and is further diluted to meet final concentration of $3 \mu \mathrm{g} / \mathrm{mL}$.

Preparation of system suitability mix: Primary stock of $0.2 \mathrm{mg} / \mathrm{mL}$ solution of Imp-B is prepared in a mixture of water: acetonitrile $50: 50 \mathrm{v} / \mathrm{v}$ for better solubility. Further diluted to make an intermediate stock of $1.5 \mu \mathrm{g} / \mathrm{mL}$ in diluent. Pipette out $1.5 \mathrm{~mL}$ of primary standard stock solution of scopolamine into a $20 \mathrm{~mL}$ volumetric flask, added $2.0 \mathrm{~mL}$ of Imp-B intermediate stock solution and rest of volume made with the diluent (Fig. 2A).

Fig. 1. Chemical structures of scopolamine and its impurities 


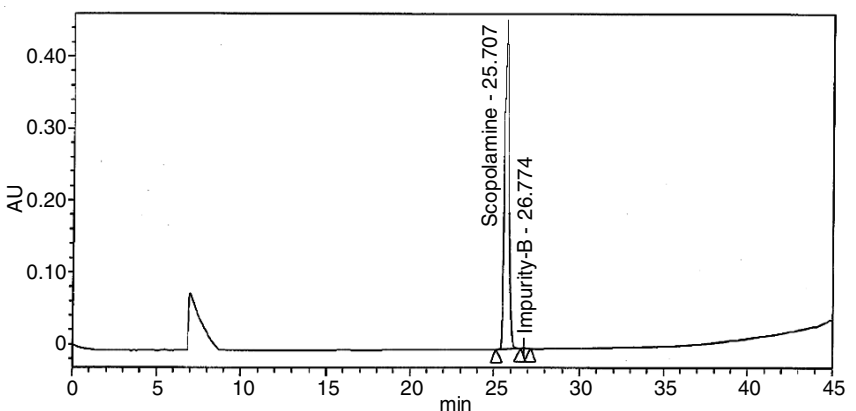

Fig. 2A. Typical chromatogram of system suitability-mix of scopolamine and Imp-B

Preparation of sample solution: Taken 2 patches containing $1.5 \mathrm{mg} / \mathrm{patch}$, peeled off the adhesive layer and transferred into a $100 \mathrm{~mL}$ conical flask added $5 \mathrm{~mL}$ of acetone, shaken on rotary shaker at $250 \mathrm{rpm}$ for $30 \mathrm{~min}$. Sonicated the flask for $60 \mathrm{~min}$ by maintaining the sonicator temperature below $15{ }^{\circ} \mathrm{C}$ with intermittent shaking. Slowly added $15 \mathrm{~mL}$ of Milli-Q water, where white precipitate appears which slowly turns to clear solution by sonication for $2 \mathrm{~h}$ with intermittent shaking and further filled in vials and freshly prepared samples were subjected for HPLC analysis (Fig. 2B).

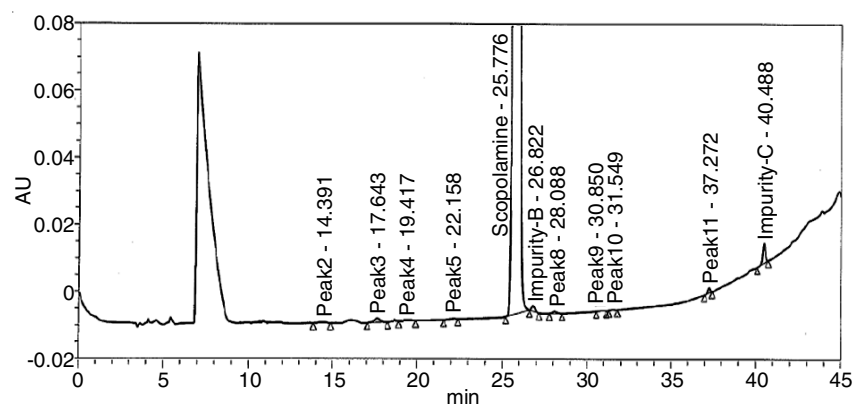

Fig. 2B. Typical chromatogram of test sample

Method development and optimization: Initial trials were performed with acidic mobile phase to understand the retentive behaviour of scopolamine and its impurities on C18 reversed phase columns. Asymmetric peaks with split pattern is noticed when trials taken with mobile phase at acidic $\mathrm{pH} 3.2$. As the $\mathrm{pKa}$ of scopolamine is 7.55-7.81, trials were initiated with $\mathrm{pH}$ 6.5 with phosphate buffer and acetonitrile as mobile phase constituents. With Xterra RP18, $250 \times 4.6 \mathrm{~mm}, 5 \mu \mathrm{m}$ column, trials were taken with linear gradient, where most trials ended up with co-elution of Imp-B with main peak (Fig. 2E). To select suitable stationary phase, columns like Chromatopak Peerless C18 $250 \times 4.6$ mm, $5 \mu \mathrm{m}$, Waters Reliant C18, $250 \times$ $4.6 \mathrm{~mm}, 5 \mu \mathrm{m}$ were screened. Upon continuous efforts, promising separationis achieved with Inertsil ODS-3V, $250 \times 4.6 \mathrm{~mm}, 5$ $\mu \mathrm{m}$, where USP resolution between Imp-B and main peak found $<$ 1.3.Refer to Fig. 2F. Hence started optimizing chromatographic conditions, where USP resolution of $>2$ is achieved between Imp-B and main peak with pH 5.5 phosphate buffer and combination of acetonitrile and methanol as organic modifiers. Satisfactory separation of all impurities were obtained with a linear curve-6 gradient of time in min/\%B: $0.0 / 5,10.0 / 5,30.0 /$ $35,40.0 / 85,40.1 / 5,45.0 / 5$ with flow rate of $0.7 \mathrm{~mL} / \mathrm{min}$ and column oven temperature of $45^{\circ} \mathrm{C}$.

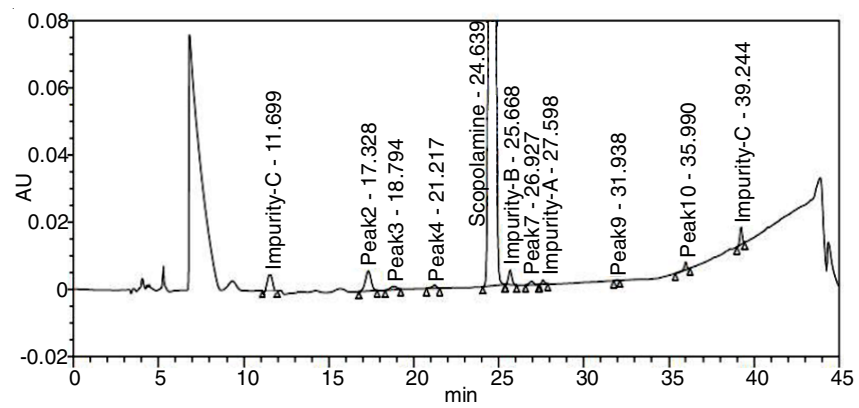

Fig. 2C. Typical chromatogram of all impurity spiked sample

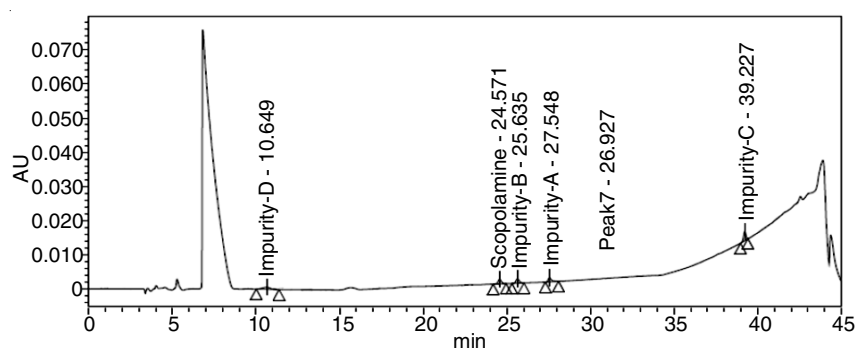

Fig. 2D. Typical chromatogram of all impurity spiked sample at LOQ level
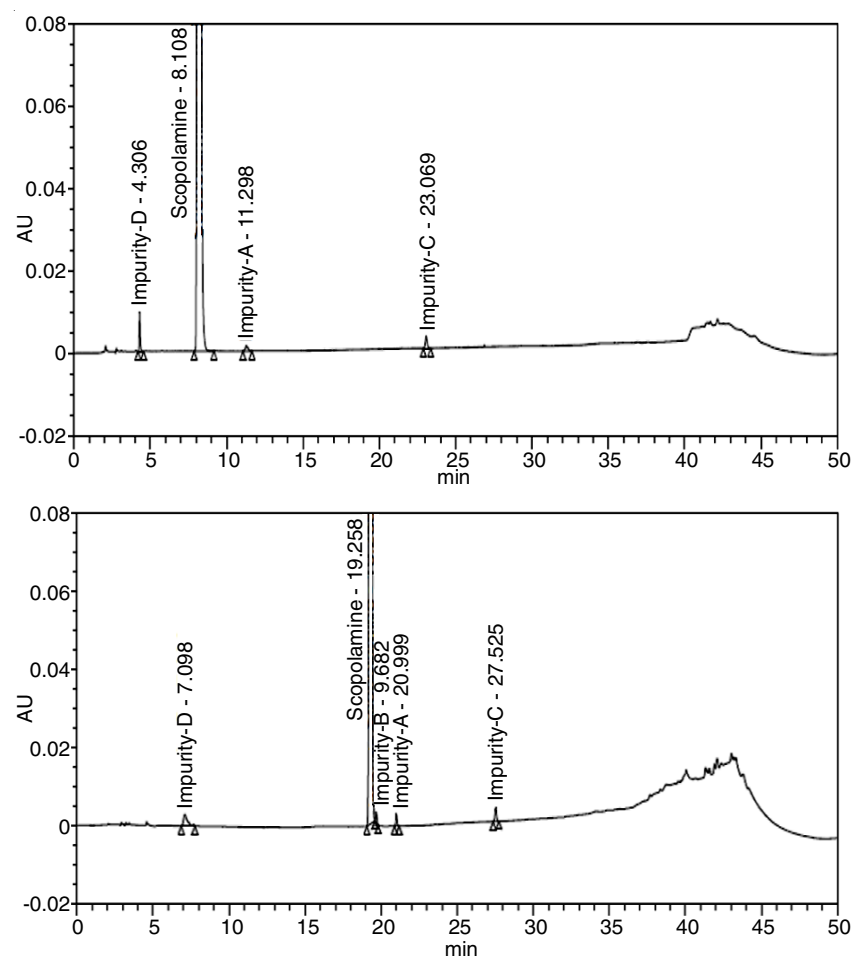

Fig. 2E and 2F. Typical chromatograms during method development (separation of Imp-B from main peak)

Once chromatographic conditions were optimized, 4 diluents were studied with combination of water with ethanol and acetonitrile, where drug is not recovered and impurities were poorly resolved with asymmetric peak shapes. With acetone upon prolonged sonication (about $60 \mathrm{~min}$ ), drug got extracted from polymer of multi-layered patch, followed by adding water and further sonication for solubility of extracted drug. Symmetric peak shape and well resolved impurities were achieved when a mixture of acetone and Milli-Q water in ratio of 10:30, $\mathrm{v} / \mathrm{v}$ is used as diluent. According to literature, under acidic and alkali stress conditions, scopolamine degrades to tropic 


\begin{tabular}{|c|c|c|c|c|c|c|}
\hline \multicolumn{7}{|c|}{$\begin{array}{c}\text { TABLE-1 } \\
\text { SUMMARY OF SYSTEM SUITABILITY AND PRECISION }\end{array}$} \\
\hline Compound & RRT & USP tailing & Precision & RSD (\%) & Inter-precision & $\operatorname{RSD}(\%)$ \\
\hline Imp-D & 0.43 & 1.3 & 0.18 & 7.4 & 0.19 & 2.6 \\
\hline Imp-B & 1.04 & 1.1 & 0.21 & 3.6 & 0.20 & 1.9 \\
\hline Imp-A & 1.12 & 1.1 & 0.22 & 1.9 & 0.21 & 3.2 \\
\hline Imp-C & 1.60 & 1.1 & 0.20 & 2.1 & 0.19 & 1.3 \\
\hline
\end{tabular}

TABLE-2

SUMMARY OF LINEARITY, LOD, LOQ, PRECISION AND RECOVERY AT LOQ

\begin{tabular}{|c|c|c|c|c|c|c|c|}
\hline \multirow{2}{*}{ Compound } & \multirow{2}{*}{$\operatorname{LOD}(\%)$} & \multirow{2}{*}{ Slope (m) } & \multirow{2}{*}{ Intercept (y) } & \multirow{2}{*}{ Correl (R) } & \multirow{2}{*}{ Bias (\%) } & \multicolumn{2}{|c|}{ LOQ precision $(n=6)$} \\
\hline & & & & & & Mean $(\%)$ & $\operatorname{RSD}(\%)$ \\
\hline Imp-D & 0.0165 & 160510.1 & -287.82 & 0.9998 & -1.686 & 0.0275 & 7.4 \\
\hline Imp-B & 0.0150 & 185607.5 & -440.01 & 0.9997 & -2.451 & 0.0250 & 2.3 \\
\hline Imp-A & 0.0152 & 171153.4 & -791.52 & 0.9997 & -4.865 & 0.0253 & 1.9 \\
\hline Imp-C & 0.0166 & 263562.5 & -1301.18 & 0.9997 & -4.679 & 0.0277 & 1.6 \\
\hline Scopolamine & 0.0158 & 172095.7 & -512.85 & 0.9997 & -2.910 & 0.0264 & 3.2 \\
\hline
\end{tabular}

Obtained USP s/n = 3 for LOD and 10 for LOQ. \% RSD for LOQ Precision is NMT $15 \%$, Recovery at LOQ should be within $85-115 \%$.

For Linearity: Correlation coefficient $>0.997$ and bias should be $\pm 5.0 \%$.

acid, which is considered as known impurity in this method, which elutes at the retention time of 11.69 min with RRT of 0.48 .

\section{RESULTS AND DISCUSSION}

Method verification: The proposed method is verified according to validation parameters of ICH guidelines [20]. Impurity blend stocks of Imp-A, Imp-B, Imp-C and Imp-D were prepared and spiked on test solution to meet $0.2 \%$ of target concentration and were used for method validation.

Precision: The precision of the method is verified by injecting six individual test preparations spiked with $0.2 \%$ of Imp-A, Imp-B, Imp-C and Imp-D. Evaluated system suitability and $\% \mathrm{RSD}$ of area for each impurity is calculated. Ruggedness of the method is determined using different instrument, column and analyst and performing the analysis on different day (Table-1, Figs. 2A, 2B and 2C).

Limits of detection (LOD) and limit of quantification (LOQ): LOD and LOQ for all four impurities, alongwith scopolamine were determined at a signal-to-noise ratio of 3:1 and 10:1, respectively, by injecting a series of dilute solutions with known concentrations. Precision at LOQ study is carried out by injecting six individual preparations of impurities and $\%$ RSD is calculated (Table-2 and Fig. 2D).

Linearity: Linearity test solutions for the method were prepared by diluting stock solution to the required concentrations. The solutions were prepared at six concentration levels from LOQ to $150 \%$ of the specification level (Table-2).

Accuracy: Accuracy of the related substance method is evaluated in triplicates using concentration levels ranging from LOQ to $150 \%$ (specification $0.2 \%$ of target concentration, i.e., $0.15 \mu \mathrm{g} / \mathrm{mL}$ ) by spiking impurities on test sample and $\%$ recovery is calculated. The same is performed with scopolamine also, to elucidate the recovery of unknown with respect to main peak (Table-3).

Robustness: Robustness of the method is evaluated by assessing the impact on system suitability parameters like USP

\begin{tabular}{ccccc}
\multicolumn{5}{c}{ TABLE-3 } \\
SUMMARY OF ACCURACY \\
\hline Compound & LOQ $(\%)$ & $50 \%$ & $100 \%$ & $150 \%$ \\
\hline Imp-D & 94.6 & 96.3 & 98.4 & 101.3 \\
Imp-B & 109.4 & 94.9 & 110.3 & 109.0 \\
Imp-A & 103.2 & 100.8 & 106.7 & 107.4 \\
Imp-C & 98.6 & 104.7 & 105.6 & 109.5 \\
Scopolamine & 98.3 & 99.4 & 104.5 & 101.7 \\
\hline \% Recovery should be within 85 to $115 \%$ for each level.
\end{tabular}

$\%$ Recovery should be within 85 to $115 \%$ for each level.

tailing and USP resolution between scopolamine and its 4 impurities by deliberately changing the chromatographic conditions. The $\mathrm{pH}$ of the mobile phase is varied from 5.3 and 5.7 (original pH: 5.5), flow rate of the method is varied from 0.6 to $0.8 \mathrm{~mL} / \mathrm{min}$ (original flow: $0.7 \mathrm{~mL} / \mathrm{min}$ ) and column oven temperature is varied from 40 to $50{ }^{\circ} \mathrm{C}$ (original temperature: $45^{\circ} \mathrm{C}$ ). Variation in mobile phase composition is performed by varying $\pm 10 \%$ of organic solvent, from original. In all the robustness studies, system suitability parameters and separation efficiency found unaltered and within acceptance limits and unaltered.

\section{Conclusion}

The newly developed gradient reversed phase LC method for quantitative analysis of scopolamine and its impurities in transdermal delivery patch formulation is found to be precise, accurate, linear and robust. Trials performed to screen suitable RP-stationary phase and extraction procedure found fit for analysis. Satisfactory results were obtained by verifying validation parameters of the method as per ICH. The method can be used for evaluating developmental, routine and stability batches of scopolamine patch.

\section{ACKNOWLEDGEMENTS}

The authors thank the Management of IPDO, Dr. Reddy's Laboratories Ltd (IP Pub No: PUB00514-17) for supporting this work. 


\section{REFERENCES}

1. S.P. Clossold and R.C. Heel, Drugs, 29, 189 (1985).

2. N. Hosseini, S.N. Ebrahimi, P. Salehi, B. Asghari and M. Ahmadi, J. Med. Plants Res., 5, 15 (2011).

3. Y. Sawabe, K. Yamasaki, T. Tagami, M. Kawaguchi, S. Taguchi, J. Nat. Med., 65, 395 (2011);

https://doi.org/10.1007/s11418-010-0483-9.

4. A. Bahmanzadegan, F. Sefidkon and A. Sonboli, Iran. J. Pharm. Res., 8, 65 (2009).

5. M.A. Fliniaux, F. Manceau and A. Jacquin-Dubreuil, J. Chromatogr. A, 644, 193 (1993);

https://doi.org/10.1016/0021-9673(93)80130-Z.

6. S. Mandal, A. Naqvi and R.S. Thakur, Phytochem. Anal., 2, 208 (1991); https://doi.org/10.1002/pca.2800020505.

7. K.H. Plank and K.G. Wagner, Z. Naturforsch. B, 41c, 391 (1986).

8. M. Masrournia, Z. Es' haghi and M. Amini, Am. J. Anal. Chem., 2, 235 (2011);

https://doi.org/10.4236/ajac.2011.22028.

9. L.J. Pennington and W.F. Schmidt, J. Pharm. Sci., 71, 951 (1982); https://doi.org/10.1002/jps.2600710830.

10. R.O. Zimmerer Jr. and L.T. Grady, J. Pharm. Sci., 59, 87 (1970); https://doi.org/10.1002/jps.2600590115.
11. J.J. Windheuser, J.L. Sutter and A. Sarrif, J. Pharm. Sci., 61, 1311 (1972); https://doi.org/10.1002/ips.2600610831.

12. M. Kartal, S. Kurucu, L. Altun, T. Ceyhan, E. Sayar, S. Cevheroglu and Y. Yetkin, Turk. J. Chem., 27, 565 (2003).

13. N.W Ali, M. Gamal, M. Abdelkawy, Pharm. Anal. Acta, S7, 006 (2013); https://doi.org/10.4172/2153-2435.S7-006.

14. P. Zhang, Y. Li, G. Liu, X. Sun, Y. Zhou, X. Deng, Q. Liao and Z. Xie, J. Sep. Sci., 37, 2664 (2014); https://doi.org/10.1002/jssc.201400534.

15. J. Beyer, F.T. Peters, T. Kraemer and H.H. Maurer, J. Mass Spectrom., 42, 621 (2007); https://doi.org/10.1002/jms.1191.

16. B. Boros, Á. Farkas, S. Jakabová, I. Bacskay, F. Kilár and A. Felinger, Chromatographia, 71 Suppl. 1, 43 (2010); https://doi.org/10.1365/s10337-010-1524-y.

17. S. Berkov and A. Pavlov, Phytochem. Anal., 15, 141 (2004); https://doi.org/10.1002/pca.756.

18. N.M. Cintron and Y.M. Chen, J. Pharm. Sci., 76, 328 (1987); https://doi.org/10.1002/jps.2600760413.

19. I.N. Papadoyannis, V.F. Samanidou, G.A. Theodoridis, G.S. Vasilikiotis, G.J.M. Van Kempen and G.M. Beelen, J. Liq. Chromatogr., 16, 975 (1993); https://doi.org/10.1080/10826079308019565.

20. ICH, Validation of Analytical Procedure: Text and Methodology Q2 (R1), In: International Conference on Harmonization (2005) 